\title{
Enhanced RAM-based Equalizers for Nonlinear Channels
}

\author{
James P. LeBlanc \\ Klipsch School of ECE \\ New Mexico State University \\ Las Cruces, NM 88003 \\ leblancenmsu.edu
}

\author{
Steven W. McLaughlin \\ School of Electrical \& Computer Eng. \\ Georgia Institute of Technology \\ Atlanta, GA \\ swmeee.gatech.edu
}

\begin{abstract}
We present a detection method for nonlinear channels with memory. This method uses a linear feedforward equalizer in conjunction with a random access memory (RAM) based (nonlinear) equalizer. The design criterion for the feedforward equalizer is non-traditional, with the intent to improve performance of the RAM-based equalizer. Development of the design criterion and a training-mode based adaptive implementation along with examples of this equalizer with a Pre-Cursor Enhanced RAM-DFE Canceller are included.
\end{abstract}

\section{Introduction}

Equalization, a method of reducing the effects of intersymbol interference (ISI) is well-known to be nonoptimal. However, maximum likelihood sequence detectors for channels with memory and/or high-order constellations may be prohibitively complex. Additionally, the required explicit knowledge of the channel parameters is often not available. This is especially true in the case of nonlinear channels considered herein. Such nonlinearities may be due to a nonlinear transmit amplifier (often used for power efficiency).

In some cases the nonlinear distortion may be minimized at the transmitter by pre-compensation techniques [1], [2], (which requires additionally transmitter complexity - and power consumption). We focus here on using receiver processing. Nonlinear channels require the use of nonlinear processing methods of which there are a variety. Classical nonlinear filters often used are the Volterra (and adaptive Volterra) filters [3], [4], for example.

However, there is also the RAM-based class of equalizers which use a finite-state machine model for the channel

- This work partially supported by New Mexico Space Grant Consortium, NASA Grant NAG 5-1491 and Sandia National Laboratories BE0236 due to the discrete nature of the channel's input in a digital communications setting. Fisher presents in [5] a RAMbased decision feedback equalizer (DFE) for the application of nonlinear magnetic recording channels called the RAMDFE.

Such RAM-based equalizers offer a low computational complexity implementation of nonlinear equalization. They are used in conjunction with linear equalizers. However, to maximize the benefits of such RAM-based techniques, while keeping memory size reasonable, attention must be given to the linear equalizer's design. We present a method of using a linear feedforward equalizer in conjunction with a RAM-based nonlinear equalizer. The design criterion of the feedforward equalizer attempts to limit the memory (timesupport) of the channel to within the limit handled by the RAM-based equalizer. This method differs from standard equalizer designs which use a "target" based method (such as the EPR4 target for magnetic recording) in that it is less constrained. This more flexible criterion can provide considerable performance improvement over minimum mean square error (MMSE) or target based designs.

\section{Problem Setting}

We focus on the bandlimited, channel containing a nonlinear amplifier such as a traveling wave tube (TWT) with additive Gaussian noise. We consider a system as in Figure 1 containing prefiltering (with impulse response $C_{\text {pres }}$ ), a TWT, and postfiltering $\left(C_{\text {post }}\right)$ chosen for the examples as

$$
C_{\mathrm{pre}}=\left[\begin{array}{c}
.9740 \\
-.1266-.1851 i \\
-.0087+.0308 i \\
.0013-.0006 i
\end{array}\right] \quad C_{\mathrm{post}}=\left[\begin{array}{c}
.0966 \\
-.2396-.3251 i \\
-.2114+.7937 i \\
.3761-.1057 i \\
-.0154-.0022 i
\end{array}\right]
$$

Here we model the TWT using the Saleh model [2] with the amplitude and phase input/output relations as shown in Figure 2. For a visual example of the nonlinear distortion 


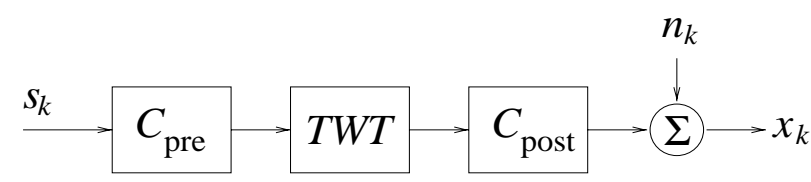

Figure 1. Nonlinear Channel Model

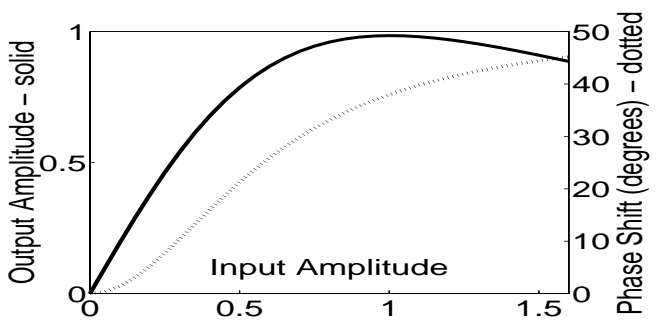

Figure 2. TWT Input/Output Characteristics

produced by this channel, see Figure 3-a which shows the noiseless channel output after equalizing with a 20-tap linear equalizer (adapted to the minimum mean-squared error (MMSE) using the least mean squares (LMS). 3-b shows again with same channel, but with the nonlinearity removed (b).

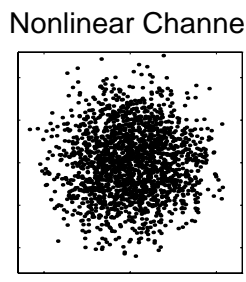

(a)

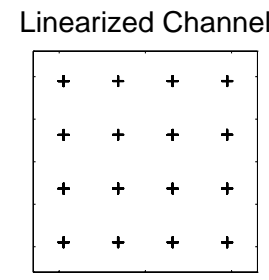

(b)
Figure 3. Linear Equalizer Output for Nonlinear Channel, and Linearized Version

\section{RAM-Based Equalizers}

In the RAM-DFE equalizer the linear transversal filter found in the feedback portion of a traditional DFE is replaced by a RAM. The bit patterns associated with past decisions form the address lines, accessing particular data which is meant to remove the (arbitrary nonlinear) ISI. The proper RAM data content is dependent on the channel and is acquired through adaptive means using training. As with all standard DFE's however, the RAM-DFE handles only post-cursor ISI terms. For this reason, linear feedforward equalizers are used in concert with it. Note that the linear feedforward equalizer is capable of attacking only the linear components of the pre-cursor ISI terms. An enhancement to the RAM-DFE, known as the Pre-Cursor Enhanced RAMDFE/Canceller (PERC) [6] was introduced with the goal of reducing both the linear and nonlinear components of the precursor ISI.

\subsection{The PERC}

The basic structure PERC equalizer is seen to be an extension of the RAM-DFE and is shown in Figure 4 where $D_{k}$ is a feedforward equalizer, and RAM denotes a random access memory with address lines consisting of $B$ past decision $\hat{s}_{k-1}$ to $\hat{s}_{k-B}$ (denoted post-address) and $A$ present/future potential decisions $\tilde{s}_{k}$ to $\tilde{s}_{k+A-1}$. We denote such a configuration as $\operatorname{PERC}(A, B)$. The PERC's twist on the RAM-DFE is the incorporation of present and future decision (to be discussed). Note that the RAM address space grows exponentially with $A+B$. (For example with $A+B=5$, for 16-QAM more then $10^{6}$ data locations are required). Aside from the amount of RAM, $A+B$ being too large leads to excessively long training periods. In light of this, even though the PERC is designed to compensate for both pre- and post-cursor ISI, it is still desirable to use a linear feedforward equalizer to reduce RAM requirements to the extent possible. The feedforward equalizer is also used to set the proper delay (in training mode).

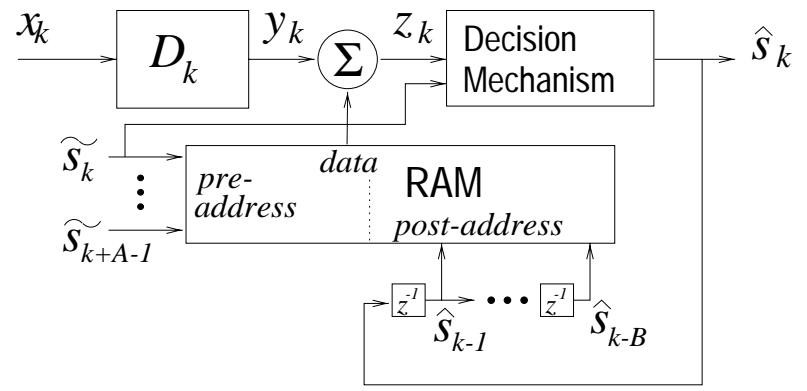

Figure 4. PERC Structure

The PERC is first run in a training mode where all "pre" $\left(\left\{\tilde{s}_{k+n-1}, \ldots, \tilde{s}_{k}\right\}\right)$ and "post" $\left(\left\{\hat{s}_{k-1}, \ldots, \hat{s}_{k-m}\right\}\right)$ components are available to learn the channel characteristics. The feedforward equalizer is trained first. One possible method would be to use the standard LMS update relation:

$$
\begin{aligned}
y_{k} & =X_{k}^{\mathrm{T}} D \\
D_{k+1} & =D_{k}-\mu_{\mathrm{ff}} X_{k}^{*}\left(y_{k}-s_{k-\Delta}\right)
\end{aligned}
$$

where $D_{k}=\left[\begin{array}{lll}d_{0} & \ldots & d_{m}\end{array}\right]^{\mathrm{T}}$ is the equalizer tap weight vector, $X_{k}=\left[\begin{array}{lll}x_{k} & \ldots & x_{k-m}\end{array}\right]^{\mathrm{T}}, \mu_{\mathrm{ff}}$ is the adaptation step-size, and $*$ denotes complex conjugation. After the delay has been set, the feedforward equalizer is then fixed and the PERC component is trained using:

$$
R A M_{k+1}(\text { add })=R A M_{k}(\text { add })-\mu_{\mathrm{fb}}\left(z_{k}-s_{k-\Delta}\right)
$$

where $R A M_{k}$ (add) denotes the contents at time $k$ of address add which is given by the bit representation of the vector $\left[\begin{array}{lllllll}\tilde{s}_{k+A-1} & \ldots & \tilde{s}_{k} & \hat{s}_{k-1} & \ldots & \hat{s}_{k-B}\end{array}\right]=$ $\left[\operatorname{add}_{\text {pre }} \mid \operatorname{add}_{\text {post }}\right]$.

After training, the PERC may be run in "fixed mode" where no adaptation takes place or in a "decision-directed" 
mode. The difficulty here is to decide what is the proper

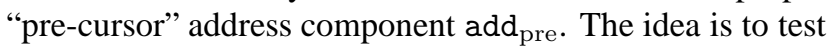
over all possible symbols of add $\mathrm{d}_{\text {pre }}$ and choose the one that places $z_{k}$ closest to a valid cursor symbol under test, $\tilde{s}_{k-\Delta}$ (i.e., the address that minimizes $\left|z_{k}-\tilde{s}_{k-\Delta}\right|^{2}$ ). Since the output is dependent on past decisions (which may be incorrect), error propagation is possible.

\section{Linear Equalizer Design Criterion}

The method chosen to determine the feedforward equalizer's tap weights will impact the overall performance. As design is difficult for nonlinear channels, we consider for the moment the linear channel problem with block diagram found in Figure 5. Here, $s_{k}$ is a zero mean, inde-

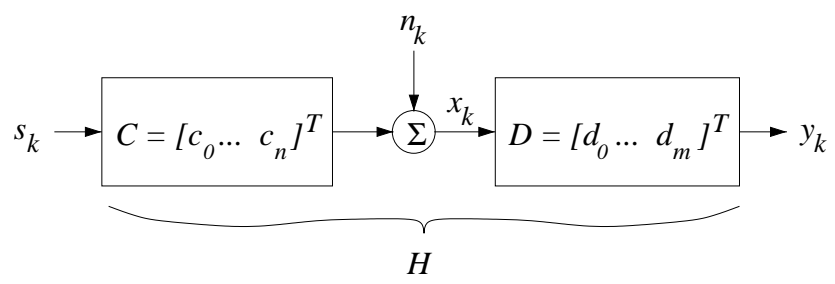

Figure 5. System Block Diagram

pendent, identically distributed (iid) source sequence, with $n_{k}$ also zero-mean iid. Defining the states of the channel and equalizer at time $k$ to be $S_{k}=\left[\begin{array}{lll}s_{k} & \ldots & s_{k-n}\end{array}\right]^{\mathrm{T}}$ and $X_{k}=\left[\begin{array}{lll}x_{k} & \ldots & x_{k-m}\end{array}\right]^{\mathrm{T}}$ respectively, we can write

$$
\begin{aligned}
& x_{k}=C^{\mathrm{T}} S_{k}+n_{k} \\
& y_{k}=D^{\mathrm{T}} X_{k}=X_{k}^{\mathrm{T}} D
\end{aligned}
$$

Denote the $(m+1) \times(n+m+1)$ channel convolution matrix as

$$
\mathbb{C}=\left[\begin{array}{ccccccc}
c_{0} & c_{1} & \ldots & c_{n} & 0 & \ldots & 0 \\
0 & c_{0} & c_{1} & \ldots & c_{n} & \ddots & \vdots \\
\vdots & \ddots & \ddots & \ddots & & \ddots & 0 \\
0 & \ldots & 0 & c_{0} & c_{1} & \ldots & c_{n}
\end{array}\right]
$$

such that $H=\left[\begin{array}{lll}h_{0} & \ldots & h_{n+m+1}\end{array}\right]^{\mathrm{T}}=\mathbb{C}^{\mathrm{T}} D$.

A standard criterion for equalizer tap weight selection is the minimum mean-square error (MMSE) criterion where the error is considered to be the distance between the equalizer output $y_{k}$ and some previous source symbol $s_{k-\Delta}$ ( $\Delta$ indicates the channel-equalizer delay), $J_{\mathrm{MMSE}}=$ $\mathcal{E}\left\{\left|y_{k}-s_{k-\Delta}\right|^{2}\right\}$. The minimum of $J_{\mathrm{MMSE}}$ is obtained by setting its gradient with respect to $D^{*}$ equal to zero, obtaining the well-known result:

$$
D_{\mathrm{opt}}=\left(\mathbb{C}^{*} \mathbb{C}^{\mathrm{T}}-\frac{\sigma_{n}^{2}}{\sigma_{s}^{2}} \mathbb{I}\right)^{-1} \widetilde{C}^{*}
$$

where $\sigma_{s}^{2}$ is the source variance, $\sigma_{n}^{2}$ is the noise variance, and $\widetilde{C}=\left[\begin{array}{lll}c_{\Delta} & \ldots & c_{\Delta-1-m}\end{array}\right]$ (assuming causality, many of the elements of $\widetilde{C}$ may be zero).

Alternative Equalizer Tap Selection Criterion. In considering the case where the equalizer is to be followed by some form of a sequence detector of limited complexity, we choose a new tap selection criterion in which there is no cost associated with intersymbol interference within the sequence detector's window.

Assuming the sequence detector is capable of handling sequences with total memory of length $L+1$, we propose the channel shortening cost function which penalizes ISI only outside of this window,

$$
J_{\mathrm{CS}}=\mathcal{E}\left\{\left|y_{k}-\left(s_{k-\Delta}+\sum_{\ell=1}^{L} h_{\ell} s_{k-\Delta-\ell}\right)\right|^{2}\right\}
$$

Note that the $L$ consecutive values of combined channelequalizer response may take on any value, without penalty nor constraint. (Also note that the arbitrary values $h_{\ell}$ can not include $\ell=0-$ this would lead to the origin being the minimum. So, introduce some constraints have been introduced). Defining

$$
S_{k}^{L}=\left[\begin{array}{c}
s_{k-\Delta-1} \\
s_{k-\Delta-2} \\
\vdots \\
s_{k-\Delta-L}
\end{array}\right], \quad H_{L}=\left[\begin{array}{c}
h_{\Delta+1} \\
h_{\Delta+2} \\
\vdots \\
h_{\Delta+L}
\end{array}\right]=\widehat{\mathbb{C}}^{\mathrm{T}} D
$$

where,

$$
\widehat{\mathbb{C}}=\left[\begin{array}{cccc}
c_{\Delta+1} & c_{\Delta+2} & \ldots & c_{\Delta+L} \\
c_{\Delta} & c_{\Delta+1} & \ldots & c_{\Delta+L-1} \\
\vdots & \vdots & \ddots & \vdots \\
c_{\Delta+1-m} & c_{\Delta+2-m} & \ldots & c_{\Delta+L-m}
\end{array}\right]
$$

we can then write the cost function in vector/matrix notation as,

$$
\begin{aligned}
J_{\mathrm{CS}} & =\mathcal{E}\left\{\left|\left(D^{\mathrm{T}} X_{k}-H_{L}^{\mathrm{T}} S_{k}^{L}\right)-s_{k-\Delta}\right|^{2}\right\} \\
& =D^{\mathrm{H}}\left(\left(\mathbb{C}^{*} \mathbb{C}^{\mathrm{T}}-\widehat{\mathbb{C}}^{*} \widehat{\mathbb{C}}^{\mathrm{T}}\right) \sigma_{s}^{2}+\sigma_{n}^{2} \mathbb{I}\right) D-2 D^{\mathrm{H}} \widetilde{C}^{*}+\sigma_{s}^{2}
\end{aligned}
$$

where ${ }^{\mathrm{H}}$ denotes the conjugate transpose (Hermitian) operator. Differentiating and equating with zero yields the optimal tap weights (under this design criterion)

$$
D_{\mathrm{opt}}=\left(\mathbb{C}^{*} \mathbb{C}^{\mathrm{T}}-\widehat{\mathbb{C}}^{*} \widehat{\mathbb{C}}^{\mathrm{T}}+\frac{\sigma_{n}^{2}}{\sigma_{s}^{2}} \mathbb{I}\right)^{-1} \widetilde{C}^{*}
$$

Continuing let $\mathbb{T}$ be a $(n+m+1) \times L$ Toeplitz matrix having its first column all zero excepts for the $\Delta+1$ entry which 
is one (the first element is considered to have index zero), then recognize that $\widehat{\mathbb{C}}=\mathbb{C} \mathbb{T}$ yielding,

$$
\begin{aligned}
D_{\mathrm{opt}} & =\left(\mathbb{C}^{*}\left(\mathbb{I}-\mathbb{T T}^{\mathrm{T}}\right) \mathbb{C}^{\mathrm{T}}+\frac{\sigma_{n}^{2}}{\sigma_{s}^{2}} \mathbb{I}\right)^{-1} \widetilde{C}^{*} \\
& =\left(\mathbb{C}^{*} \mathbb{M} \mathbb{C}^{\mathrm{T}}+\frac{\sigma_{n}^{2}}{\sigma_{s}^{2}} \mathbb{I}\right)^{-1} \widetilde{C}^{*}
\end{aligned}
$$

where $\mathbb{M}=\mathbb{I}-\mathbb{T T}^{\mathrm{T}}$ is the identity matrix with the $\Delta+1$ through $\Delta+L$ diagonal elements set to zero (which acts like a mask).

The following example contrasts the results of designing with $J_{\mathrm{MMSE}}$ and $J_{\mathrm{CS}}$. For our example channel (with the nonlinear TWT ignored for the moment), we obtain Figures 6 and 7 showing the optimum equalizer tap values and resulting channel-equalizer responses with $E_{b} / N_{0}=15 d B$.
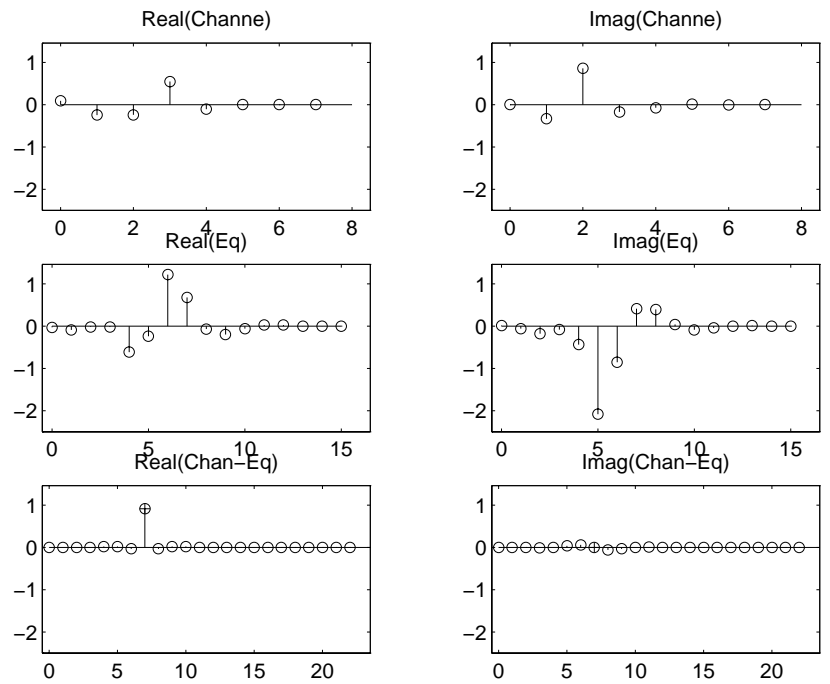

Figure 6. Optimum MMSE Eq. and Chan-Eq Response $(L=0, \Delta=7, m=15)$

In Figure $6, D_{\mathrm{MMSE}}$ appears to have virtually no ISI (for this linear channel). However, its noise gain is higher than that of the $D_{\mathrm{CS}}$ equalizer. Moreover, if one considers the ISI that is outside of the range of $h_{\Delta}$ to $h_{\Delta-L}$, then $D_{\mathrm{MMSE}}$ actually has more ISI. Through such argument, we offer an admittedly ad-hoc figure of merit:

$$
\mathrm{FOM}=\frac{\mathrm{SG}}{\mathrm{NG} \times \mathrm{ISI}}
$$

where,

$$
\begin{aligned}
\mathrm{SG} & =\sum_{i=\Delta}^{\Delta+L}\left|h_{i}\right|^{2}=H_{L}^{\mathrm{H}} H_{L} \\
\mathrm{NG} & =\sum_{i=0}^{m}\left|d_{i}\right|^{2}=D^{\mathrm{H}} D
\end{aligned}
$$
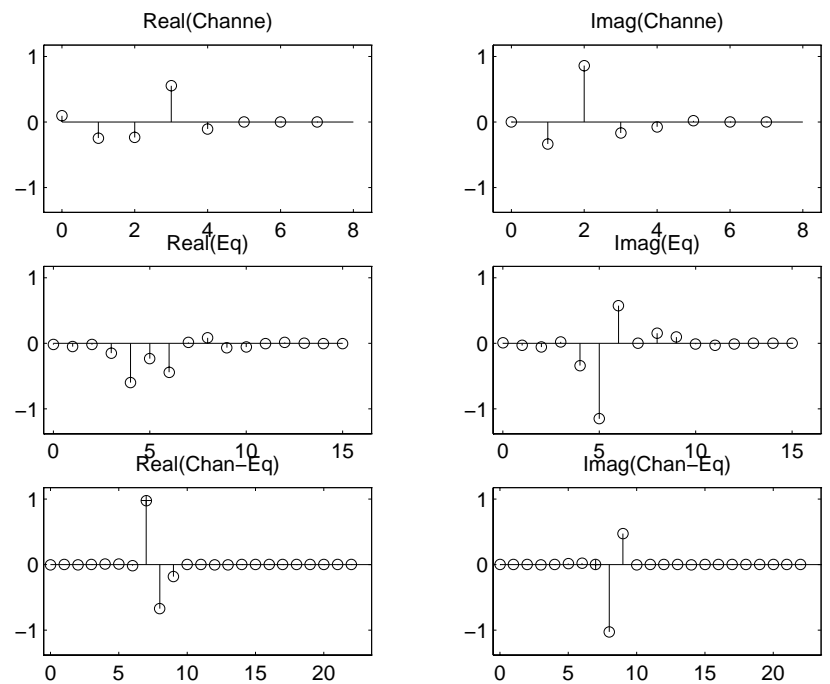

Figure 7. Optimum CS Eq. and Chan-Eq Response $(L=2, \Delta=7, m=15)$

\begin{tabular}{|c|c|ccc|}
\hline & FOM (dB) & SG (dB) & NG (dB) & ISI (dB) \\
\hline$J_{\text {MMSE }}$ & 32.2 & 0.8 & -11.9 & -18.3 \\
\hline$J_{\text {CS }}$ & 49.6 & 4.3 & -17.1 & -28.2 \\
\hline
\end{tabular}

Table 1. Comparison of Metrics for MMSE and CS designs

$$
\mathrm{ISI}=\sum_{i=0}^{\Delta-1}\left|h_{i}\right|^{2}+\sum_{i=L+1}^{n+m+1}\left|h_{i}\right|^{2}
$$

The basic idea is to consider the power of the signal entering the sequence detector $(\mathrm{SG})$ to be a useful quantity, while the noise gain (NG) and isi outside of the detectors "window" (ISI) have deleterious effects. These quantities from the above example are found in Table 1. (It should be pointed out that the $17 \mathrm{~dB}$ difference in FOM should not be expected to result in a similar $\mathrm{dB}$ improvement in other metrics (i.e. MSE or bit error rate, etc.) The point remains, that $J_{\mathrm{CS}}$ results in less noise gain, less ISI outside of the detector's window, and more signal power into the detector.

Adaptive Implementation of CS criterion. We return to considering the nonlinear channel. In this case (or even in linear channel cases where $C$ is unknown), one cannot design directly to channel shortening criterion. However, in a manner similar to the LMS adaptive algorithm, one can follow a gradient descent path based on the instantaneous gradient and averaging $J_{\mathrm{CS}}$ with

$$
\begin{aligned}
\nabla_{\mathrm{D}} J_{\mathrm{CS}} & =X_{k}^{*} X_{k}^{\mathrm{T}} D-C^{*} T S_{k}^{\mathrm{L} *} X_{k}^{\mathrm{T}} D-X_{k}^{*} s_{k-\Delta} \\
& =X_{k}^{*}\left(y_{k}-s_{k-\Delta}\right)-\left(S_{k}^{\mathrm{L} *} X_{k}^{\mathrm{T}}\right)^{\mathrm{H}}\left(S_{k}^{\mathrm{L} *} X_{k}^{\mathrm{T}}\right) D
\end{aligned}
$$

where some averaging must be applied to the $S_{k}^{\mathrm{L} *} X_{k}^{\mathrm{T}}$ terms. We implement a simple AR averaging scheme. Let $\Phi_{k}^{\mathrm{T}}$ rep- 
resent such an averaged version of $S_{k}^{\mathrm{L} *} X_{k}^{\mathrm{T}}$, then we have $\Phi_{k}^{\mathrm{T}} \approx \widehat{C}^{\mathrm{T}}$.

we have the adaptive version of $J_{C S}$ minimization as,

$$
\begin{aligned}
\Phi_{k+1} & =\alpha \Phi_{k}+(1-\alpha) X_{k}^{*} S_{k}^{L \mathrm{H}} \\
y_{k} & =X_{k}^{\mathrm{T}} D_{k} \\
D_{k+1} & =D_{k}-\mu_{\mathrm{ff}} X_{k}^{*}\left(y_{k}-s_{k-\Delta}\right)-\Phi^{*} \Phi^{\mathrm{T}} D_{k}
\end{aligned}
$$

It appears that local may exist with this adaptive version, as indicated at by our simulations, but no analysis has yet been performed. However, all minima witnessed to do conform to the memory shortening constraint resulting in similarly better performance in concert with the PERC with the nonlinear channel than the standard LMS (i.e. standard MMSE) solutions.

\subsection{Simulation Examples}

For a variety of signal to noise ratios $\left(E_{b} / N_{0}\right)$ simulations were run using the nonlinear channel given by $C_{\text {pre }}$ and $C_{\text {post }}$ with a $\operatorname{PERC}(2,2), \Delta=7, m=15$. Figures 8 and 9 illustrate the constellations of 16-QAM signals at various point in the signal path for both the MMSE and CS adaptive algorithms. Figure 10 shows the final MSE performance after convergence over a range of SNR for the cases $L=0$ (MMSE), and $L=1$ and $L=2$ (CS). It is interesting to view the MSE trajectories during the training process (Figure 11) between the MMSE and CS adaptations. Feedforward equalizer adaptation is performed over blocks 1-50 and PERC adaptation from 51-150. The MMSE method has lower MSE when only a linear equalizer is used. However, when the PERC is switched on, the CS methods achieves lower MSE very quickly.
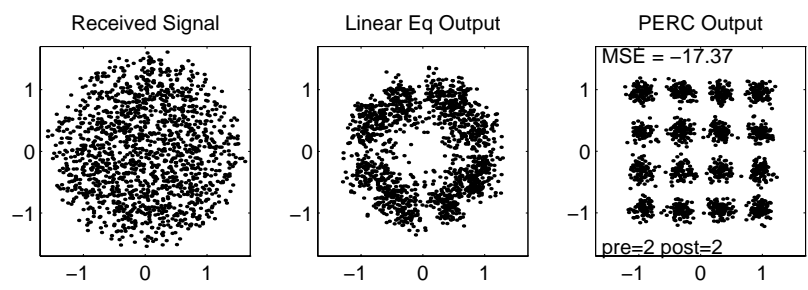

Figure 8. MMSE Constellations $\left(E_{b} / N_{0}=15 \mathrm{~dB}\right)$
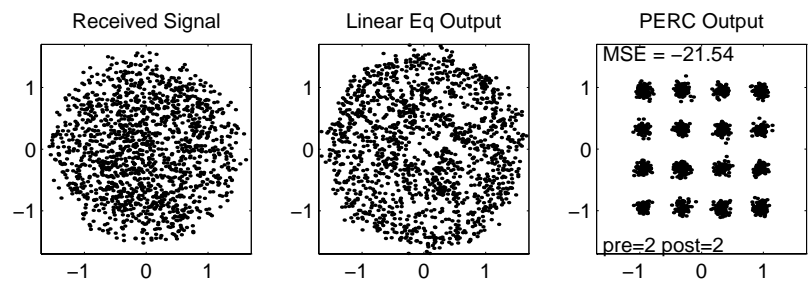

Figure 9. CS Constellations $\left(E_{b} / N_{0}=15 \mathrm{~dB}\right)$

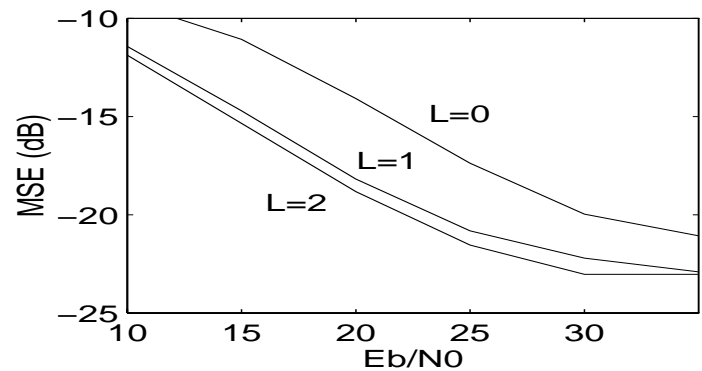

Figure 10. MSE for various values of $L$

MSE vs.TIME

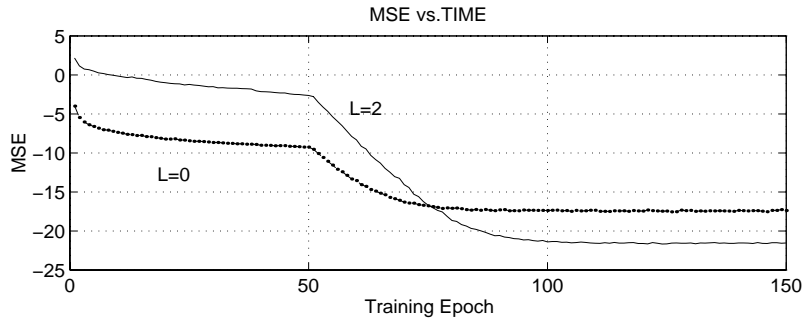

Figure 11. MSE trajectory comparison for MMSE and CS criterion

\section{References}

[1] G. Karam, H. Sari, "Analysis of Predistortion, and ISI cancellation Techniques in Digital Radio Systems with Nonlinear Transmit Amplifiers," IEEE Trans. on Comm., vol. 37, no. 12, Dec. 1989.

[2] A. A. M. Saleh, J. Salz, "Adaptive Linearization of Power Amplifiers in Digital Radio Systems," Bell Sys. Tech. Journal, vol. 62, no. 4, Apr. 1983.

[3] S. Benedetto, E. Biglieri, "Nonlinear Equalization of Digital Satellite Channels," IEEE Journal on Sel. Areas in Comm., vol. SAC-1, no. 1, Jan. 1983.

[4] E. Biglieri, A. Gersho, R. D. Gitlin, T. L. lim, "Adaptive Cancellation of Nonlinear Intersymbol Interference for Voiceband Data Transmission," IEEE Journal on Sel. Areas in Comm., vol. SAC-2, no. 5, Sept. 1984.

[5] K. D. Fisher, J. M. Cioffi, W. L. Abbot, S. Bednarz,C. M. Melas, "An Adaptive RAM-DFE for Storage Channels," IEEE Trans. on Comm., vol. 39, no. 11, Nov. 1991.

[6] J. P. LeBlanc, R. A. Kennedy, "Pre-Cursor Extended RAM-DFE Canceller (PERC) Nonlinear Equalizer for Nonlinear ISI Channels," IEEE DSP Workshop, Aug. 1998. 